Algebraic $8 \mathcal{G}$ Geometric $\mathcal{T}_{\text {opology }}$

Volume 4 (2004) 935-942

Published: 13 October 2004

ATG

\title{
Whitehead doubling persists
}

\author{
Stavros Garoufalidis
}

\begin{abstract}
The operation of (untwisted) Whitehead doubling trivializes the Alexander module of a knot (and consequently, all known abelian invariants), and converts knots to topologically slice ones. In this note we show that Whitehead doubling does not trivialize the rational function that equals to the 2-loop part of the Kontsevich integral.
\end{abstract}

AMS Classification 57N10; 57M25

Keywords Whitehead double, loop filtration, Goussarov-Habiro, clovers, claspers, Kontsevich integral

\section{Introduction}

\section{$1.1 \quad$ History}

The colored Jones function $J$ of a knot $K$ is a 2-parameter formal power series

$$
J(K)(h, \lambda)=\sum_{n, m=0}^{\infty} a_{n, m}(K) h^{n} \lambda^{m}
$$

with remarkable periodicity properties. By its definition, if $\lambda=d$ is a natural number, $J(h, d)$ coincides with the colored Jones polynomial of the knot (using the $(d+1)$-dimensional irreducible representation of $\left.\mathfrak{s l}_{2}\right)$ and thus

$$
J(h, d) \in \mathbb{Z}\left[e^{ \pm h}\right] .
$$

We can think of this as a periodicity property (i.e., a set of recursion relations) for the coefficients $a_{n, m}$ of $J$. This is an obvious periodicity property.

We now come to describe some hidden periodicity of $J$. Each coefficient $a_{n, m}$ is a Vassiliev invariant of degree $n$ and vanishes if $m>n$. Thus, we can rearrange $J$ as a sum of subdiagonal terms

$$
J(h, \lambda)=\sum_{k=0}^{\infty} h^{k} Q_{J, k}(h \lambda)
$$


where

$$
Q_{J, k}(s)=\sum_{m=0}^{\infty} a_{k+m, m} s^{m} .
$$

The MMR conjecture, shown in [BG], states that $Q_{J, 0}$ is a reparametrization of the Alexander polynomial of the knot. This translates to a hidden periodicity of $J$.

Rozansky, in his study of the colored Jones function conjectured that for every $n \geq 1, Q_{n}$ is a reparametrization of a rational function, whose denominator is a power of the Alexander polynomial. Using a variety of quantum field theory techniques and an appropriate expansion of the $R$-martix, in a difficult paper Rozansky proved the above mentioned conjecture, Ro1].

It is well-known that the colored Jones function is an image of the graph-valued universal Vassiliev invariant, the so-called Kontsevich integral. The Kontsevich integral has a subdiagonal expansion, and Rozansky further conjectured that each term of the expansion of the Kontsevich integral should be given by a series of trivalent graphs with rational functions attached to their edges. This is often called the Rationality Conjecture.

A weak form of the Rationality Conjecture was quickly proven by Kricker $\mathrm{Kr}$. A stong form followed by joint work with Kricker [GK], where a rational noncommutative invariant $Z^{\text {rat }}$ of knots was constructed.

Although the construction of the $Z^{\text {rat }}$ invariant is rather involved, there are several axiomatic properties which make it easier to understand parts of the $Z^{\text {rat }}$ invariant in terms of geometric invariants of knots.

For example, consider the move that replaces a knot $K$ in an integer homology sphere by a knot $K^{\prime}$ obtained by surgery on a hullhomologous clasper in the complement of $K$. This null-move was introduced in GR. For a reference on claspers, see Gu1, Gu2, $\mathrm{Ha}$ and also GGP. As was shown in GK], each term of the $Z^{\text {rat }}$ is a finite type invariantwith respect to the null move. Moreover, 0 -equivalence (under the null move) coincides with $S$-equivalence. Below, we will evaluate $Z^{\text {rat }}$ on a set of $S$-equivalent knots. In this case, the matrix part of $Z^{\text {rat }}$ is fixed and its graph part takes values in a graded vector space, see GK]. Let $Q_{n}=Z_{n}^{\text {rat }}$ denote the degree $n$ part of $Z^{\text {rat }}$.

$Q_{n}$ takes values in a vector space over $\mathbb{Q}$ generated by trivalent graphs with $2 n$ trivalent vertices. The graphs have rational functions in $t$ assigned to their edges, and satisfy certain linear relations explained in [GR] and GK]. For the statement and the proof of Theorem 1 below, we only need to know that $Q_{n}$ takes value in an abelian group; and any vector space over $\mathbb{Q}$ is an abelian group. 


\subsection{Statement of the results}

Our first result concerns the change of $Q_{n}$ under a modification of a knot. All knots will be oriented and, unless otherwise mentioned, 0 -framed. Consider a knot $K_{0}$ which intersects a ball $B \subset S^{3}$ in two unknotted arcs with opposite orientation. This pattern $\mathfrak{p}=\left(K_{0}, B\right)$ gives rise to a map:

$$
\tau_{\mathfrak{p}}: \text { Knots } \rightarrow \text { Knots }
$$

which sends $K$ to the result of replacing $B \cap K_{0}$ with a 2-parallel of a 1-tangle version of $K$, with 0 -framing.

This move on knots can be described in terms of surgery in the ambient space and has a long history. It was already used in the sixties (under the term, surgical modification, see for example Levine [L1]) to prove realization theorems for algebraic obstructions. Modification of knots was also used in the seventies by Casson-Gordon in their secondary obstruction invariants. Later on, the theme was taken by many authors including Gilmer, Livingston, and CochranOrr-Teichner (the latter, use the biological term: infection). In the world of finite type invariants, a systematic study of geometric surgery on clovers or claspers was initiated by Goussarov and Habiro Gu1, Ha.

Theorem 1 (i) For fixed $K_{0}$ and $n \geq 1$, the map

$$
\phi_{n}: K \rightarrow Q_{n}\left(\tau_{\mathfrak{p}}(K)\right)
$$

is a finite type invariant of 0 -framed knots of type $2 n$, whose degree $2 n$ part lies in the algebra of Alexander-Conway coefficients.

(ii) For $n=1$, we have that

$$
Q_{1}\left(\tau_{\mathfrak{p}}(K)\right)=Q_{1}\left(\tau_{\mathfrak{p}}(\text { unknot })\right)+c_{\mathfrak{p}} \cdot a(K)
$$

where $a(K)$ is a nontrivial Vassiliev invariant of degree 2 (such as the second derivative of the Alexander polynomial) and $c_{\mathfrak{p}}$ is a constant that depends on the pattern.

As an application, consider the following pattern:

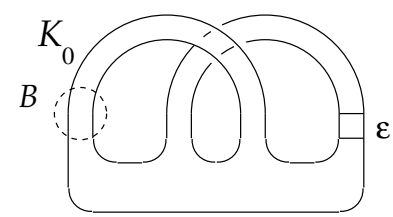

(where the $\varepsilon= \pm 1$ indicates a full twist, depending on the sign of the clasp). Then,

$$
\tau_{\mathfrak{p}}(K)=\mathrm{Wh}^{\epsilon}(K)
$$


is the untwisted Whitehead double of $K$ with either clasp, i.e., the satellite of $K$ with respect to the pattern:

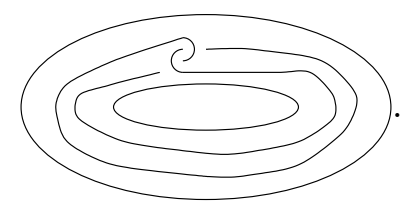

Here is a sample corollary. Since it involves explicit computations with the $Q_{1}$ invariant evaluated on knots with trivial Alexander polynomial, we need to recall that

$$
Q_{1}: \text { Knots with trivial Alexander polynomial } \rightarrow \mathcal{A}_{2}(\Lambda)
$$

where

$$
\mathcal{A}_{2}(\Lambda)=\otimes^{3} \mathbb{Q}\left[t^{ \pm 1}\right] /((f, g, h)=(t f, t g, t h), \operatorname{Aut}(\Theta))
$$

is the quotient of the abelian group $\otimes^{3} \mathbb{Q}\left[t^{ \pm 1}\right]$ modulo the subgroup generated by $a \otimes b \otimes c=t a \otimes t b \otimes t c$ and the subgroup generated by $a \otimes b \otimes c=g a \otimes g b \otimes g c$ for $g \in$ Aut $(\Theta)$. Here, Aut $(\Theta)=\operatorname{Sym}_{3} \times \operatorname{Sym}_{2}$ which acts on $\otimes^{3} \mathbb{Q}\left[t^{ \pm 1}\right]$ by permutation on the 3 factors of the tensor product and by simultaneous replacement of $t$ (in all factors of the tensor product) by $t^{-1}$.

In order to make contact with [GR] and [GK, Sec.5.3], and to explain the origin of the $\operatorname{Aut}(\Theta)$ symmetry, we point out a graphical interpretation of elements of $\mathcal{A}_{2}$ by trivalent graphs with oriented edges and beads (that is, elements of $\left.\mathbb{Q}\left[t^{ \pm 1}\right]\right)$ on their edges:

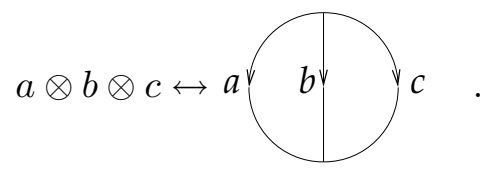

Corollary 1.1 We have:

$$
Q_{1}\left(\mathrm{Wh}^{\epsilon}(K)\right)=\varepsilon a(K) \cdot 1 \otimes 1 \otimes\left(t+t^{-1}-2\right)
$$

where $a(K)=1 /\left.4 \frac{d^{2}}{d h^{2}}\right|_{h=0} \Delta(K)\left(e^{h}\right) \in \mathbb{Z}$, and $\varepsilon= \pm 1$ is the sign of the clasp.

Whitehead doubling trivializes the Alexander module as well as the more sophisticated topological slicing obstructions of Casson-Gordon and Cochran-OrrTeichner. On the other hand, $Q_{1}$ remembers better the knot that is about to be Whitehead doubled. 


\section{Proofs}

\section{Proof of Theorem 1}

Let us recall three different moves on the set $\mathcal{K}$ of 0 -framed knots in $S^{3}$ :

- Changing a crossing, i.e., doing an I-modification in the language of Gu2 and $\mathrm{Ha}$.

- Doing a $\Delta$-move, i.e., doing a $Y$-modification in the language of $[\mathrm{MN}]$.

- Doing a null-move, in the language of GR.

These three moves lead in the usual way to three notions of finite type invariants and corresponding notions of $n$-equivalence, denoted by $\equiv_{n}^{I}, \equiv_{n}^{Y}$ and $\equiv_{n}^{l}$ respectively. Note that $K \equiv_{n} K^{\prime}$ implies that $f(K)=f\left(K^{\prime}\right)$ for all invariants $f$ of type $n$. It is a folk result (easily proven by the results of Goussarov and Habiro) that $K \equiv_{n+1}^{I} K^{\prime}$ iff $K \equiv_{n}^{Y} K^{\prime}$. Furthermore, it is easy to see that if $K \equiv_{n}^{Y} K^{\prime}$, then $\tau_{\mathfrak{p}}(K) \equiv_{n}^{l} \tau_{\mathfrak{p}}\left(K^{\prime}\right)$. Further, in [GR] and [GK], itt was shown that $Q_{n}$ is a finite type invariant of type $2 n$ with respect to the null move, where $Q_{n}$ takes values in an appropriate $\mathbb{Q}$-vector space.

This discussion implies the following conclusion, for every fixed $n$.

$$
K \equiv_{2 n+1}^{I} K^{\prime} \Longrightarrow K \equiv_{2 n}^{Y} K^{\prime} \Longrightarrow \tau_{\mathfrak{p}}(K) \equiv_{2 n}^{l} \tau_{\mathfrak{p}}\left(K^{\prime}\right) \Longrightarrow \phi_{n}(K)=\phi_{n}\left(K^{\prime}\right) .
$$

Thus, $\phi_{n}: K \mapsto Q_{n}\left(\tau_{\mathfrak{p}}(K)\right)$ is an additive (under connected sum) function on $\mathcal{K} / \mathcal{K}_{2 n+1}^{I}$ (here $\mathcal{K}_{n}^{I}$ denotes the set of $n$-trivial knots with respect to the $I$ move). By a theorem of Goussarov and Habiro it follows that $\phi_{n}$ is a Vassiliev invariant of degree at most $2 n+1$. We claim that $\phi_{n}$ is $\mathbb{Q}$-valued of Vassiliev degree $2 n$. Indeed, $\mathcal{K}_{2 n}^{I} / \mathcal{K}_{2 n+1}^{I} \otimes \mathbb{Q}$ is a vector space spanned by uni-trivalent graphs $G$ with $2(2 n+1)$ vertices such that every component of $G$ contains a trivalent vertex, modulo the AS and IHX relations. Using the AS relation, we can assume that distinct univalent vertices of $G$ are joined to distinct trivalent vertices. It follows that the number of trivalent vertices of $G$ is at least equal to the number of univalent vertices, and thus that $G$ has at least $2 n+1$ trivalent vertices, which gives rise to a null move of degree $2 n+1$, on which $Q_{n}$ vanishes.

This implies that $\phi_{n}$ is of Vassiliev degree $2 n$. Furthermore, if $G$ is a unitrivalent graph of degree $2 n$ with more than $2 n$ trivalent vertices, then $\phi_{n}(G)=0$. The remaining graphs of degree $2 n$ are a disjoint union of wheels i.e., diagrams like - This, together with a result of Kricker-Spence-Aitchinson [KSA] implies that the degree $2 n$ part of $Q_{n}$ lies in the algebra of Alexander-Conway coefficients. 
This concludes the proof of the first part Theorem 1. The second part follows easily since, up to a multiple, there is a unique $\mathbb{Q}$-valued Vassiliev invariant of $I$-degree 2 .

\section{Proof of Corollary 1.1}

Notice that $\mathrm{Wh}^{\epsilon}$ (unknot) $=$ unknot and that $Q_{1}$ (unknot) $=0$. Thus, $\phi_{1}(K)=$ $c a(K)$, where $a(K)=\left.\frac{d^{2}}{d t^{2}}\right|_{t=1} \Delta(K)(t)$ and $c$ is a constant. In order to figure out $c$, we need a computation. Let $G=\left(G_{1}, G_{2}\right)$ be a wheel with two legs attached to an unknot $K$. Consider the Y-link of degree 2 (also denoted by $G$ ) in the complement of $\mathrm{Wh}^{\epsilon}(K)$. Let $L_{i j}$ for $i=1,2$ and $j=1,2,3$ denote the six leaves of $G$ labeled as in Figure 1
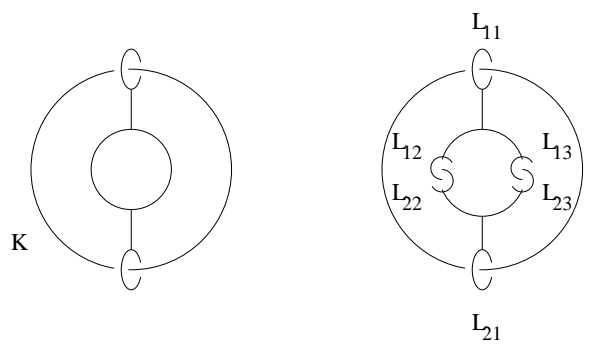

Figure 1: On the left, a wheel with two legs. On the right, the associated clover of degree 2

It follows from GR (see also GK]) that $Q_{1}\left(\left[\left(S^{3}, \mathrm{Wh}^{\epsilon}(K)\right), G\right]\right)$ is the result of complete contractions of all pairs of leaves of $G_{1} \cup G_{2}$, where we label the edges by equivariant linking numbers of the leaves. This gives rise to a linear combination of trivalent graphs with two trivalent vertices and edges decorated by elements of $\mathbb{Z}\left[t, t^{-1}\right]$.

The equivariant linking function $\mathrm{lk}_{\widetilde{M}}^{\gamma}$ (see [GR] ) of the leaves is given as follows:

$$
\mathrm{lk}_{\widetilde{M}}^{\gamma}\left(L_{1 i}, L_{2 j}\right)= \begin{cases}\delta_{i, j} & \text { for } i=2,3 \\ \varepsilon \delta_{1, j}\left(t+t^{-1}-2\right) & \text { for } i=1,\end{cases}
$$

where $\delta_{a, b}=1$ (resp. 0) if $a=b$ (resp. $a \neq b$ ), and the sign $\varepsilon= \pm 1$ depends on the sign of the clasp of the Whitehead double.

The computation of the equivariant linking function is best seen by drawing the universal abelian cover of $\mathrm{Wh}^{\epsilon}(K)$ and lifting $G$ to it.

Alternatively, one may use the genus 1 Seifert surface of $\mathrm{Wh}^{\epsilon}(K)$ drawn above and identify the equivariant linking numbers in question with the equivariant linking numbers of links formed by meridians dual to the bands. 
In [L2, Prop.14.3] Levine computes the matrix $B=\left(\operatorname{lk}_{\widetilde{M}}^{\gamma}\left(m_{i}, m_{j}\right)\right)_{i, j}$ of equivariant linking numbers of meridians $m_{i}$ dual to the bands of a Seifert surface by:

$$
B=(t-1)\left(t A-A^{T}\right)^{-1}
$$

where $A$ is the Seifert matrix with respect to a basis consisting of bands, and $A^{T}$ is the transpose of $A$. In our case, the Seifert matrix is

$$
A=\left(\begin{array}{ll}
0 & 1 \\
0 & \varepsilon
\end{array}\right)
$$

and the corresponding matrix $B$ is

$$
A=\left(\begin{array}{cc}
\varepsilon\left(t+t^{-1}-2\right) & 1-t \\
1-t^{-1} & 0
\end{array}\right) \text {. }
$$

On the other hand, we have that $a([K, G])=1$, since

$$
\Delta(K)\left(e^{h}\right)=\exp \left(-2 \sum_{n} a_{2 n}(K) h^{2 n}\right),
$$

where $a_{2 n}(K)$ is the coefficient of the degree $2 n$ wheel $w_{2 n}$ in the logarithm of the Kontsevich integral, $\mathrm{KSA}$. The result follows.

Acknowledgments The author is partially supported by NSF grant DMS98-00703 and by an Israel-US BSF grant.

\section{References}

[BG] D. Bar-Natan, S. Garoufalidis, On the Melvin-Morton-Rozansky conjecture, Inventiones Math. 125 (1996) 103-133. MathReview

[COT] T. Cochran, K. Orr and P. Teichner, Knot concordance, Whitney towsers and $L^{2}$ signatures, Annals of Math. 157 (2003) 433-519. MathReview

[F] M. Freedman, A new technique for the link slice problem, Inventiones Math. 80 (1985) 453-465. MathReview

[GGP] S. Garoufalidis, M. Goussarov and M. Polyak, Calculus of clovers and finite type invariants of 3-manifolds, Geometry and Topology, 5 (2001) no3 75-108. MathReview

[GK] S. Garoufalidis and A. Kricker, A rational noncommutative invariant of boundary links, Geom. Topol. 8 (2004) 115-204. MathReview 
[GR] S. Garoufalidis and L. Rozansky, The loop expansion of the Kontsevich integral, abelian invariants of knots and $S$-equivalence, Topology, 43 (2004) 1183-1210. MathReview

[Gu1] M. Goussarov, Finite type invariants and n-equivalence of 3-manifolds, C. R. Acad. Sci. Paris Ser. I. Math. 329 (1999) 517-522. MathReview

[Gu2] M. Goussarov, Variations of knotted graphs. The geometric technique of $n$ equivalence, St. Petersburg Math. J. 12 (2001) 569-604. MathReview

[Ha] K. Habiro, Clasper theory and finite type invariants of links, Geom. Topol. 4 (2000) 1-83. MathReview

[KSA] A. Kricker, B. Spence, I. Aitchinson, Cabling the Vassiliev invariants, J. Knot Theory and its Ramifications 6 no. 3, (1997) 327-358. MathReview

[Kr] A. Kricker, The lines of the Kontsevich integral and Rozansky's Rationality Conjecture, arXiv:math.GT/0005284.

[L1] J. Levine, A characterization of knot polynomials, Topology 4 (1965) 135-141. MathReview

[L2] J. Levine, Knot modules I, Transactions of the AMS, 229 (1977) 1-50. MathReview

[MN] H. Murakami and Y. Nakanishi, On a certain move generating link homology, Math. Annalen 284 (1989) 75-89. MathReview

[Rf] D. Rolfsen, A surgical view of Alexander's polynomial, in Geometric Topology (Utah 1974), Lecture Notes Math. 438 Springer-Verlag, 1974, 415-423. MathReview

[Ro1] L. Rozansky, The universal R-matrix, Burau Representation and the MelvinMorton expansion of the colored Jones polynomial, Adv. Math. 134 (1998) 1-31. MathReview

[Ro2] L. Rozansky, A rationality conjecture about Kontsevich integral of knots and its implications to the structure of the colored Jones polynomial, Proceedings of the Pacific Institute for the Mathematical Sciences Workshop "Invariants of ThreeManifolds" (Calgary, 1999). Topology Appl. 127 (2003) 47-76. MathReview

School of Mathemtaics, Georgia Institute of Technology

Atlanta, GA 30332-0160, USA.

Email: stavros@maths.gatech.edu

URL: http://www.math.gatech.edu/^stavros

Received: 27 March $2001 \quad$ Revised: 27 September 2004 